\title{
Editorial \\ Dysautonomia, fibromyalgia and reflex dystrophy
} Jean Eisinger

Unit Infomyalgies, Centre Hospitalier, 83056 Toulon, France

Corresponding author: Jean Eisinger, infomyalgies@wanadoo.fr

Published: 6 July 2007

Arthritis Research \& Therapy 2007, 9:105 (doi:10.1186/ar2212)

This article is online at http://arthritis-research.com/content/9/4/105

(C) 2007 BioMed Central Ltd

See related review by Martinez-Lavin, http://arthritis-research.com/content/9/4/216

\begin{abstract}
Autonomic nervous system dysfunction observed in fibromyalgia, characterized without exception by a sympathetic hyperactivity and hyporeactivity, has been reported. However, several studies demonstrated reduced levels of norepinephrine and neuropeptide $Y$ at rest and after tilt table in some patients, which was improved by beta-stimulating agents. These findings support heterogeneity in fibromyalgia-associated dysautonomia. Fibromyalgia could be a generalized sympathetic dystrophy since both conditions are activated by trauma and partly linked to sympathetic mechanisms. Yet they differ on several points: hormonal and neurochemical abnormalities are observed in fibromyalgia whereas activation by peripheral trauma and hyperosteolysis are observed in reflex sympathetic dystrophy.
\end{abstract}

In a well documented review on the stress response system, Martinez-Lavin [1] proposes an original approach to dysautonomia, fibromyalgia syndrome (FMS) and reflex sympathetic dystrophy (RSD). Several points are probably oversimplified and deserve some comments, based on two questions: is the autonomic nervous system dysfunction observed in FMS the same for all patients? And is fibromyalgia a generalized sympathetic dystrophy?

\section{Is the autonomic nervous system dysfunction observed in FMS the same for all patients?}

Martinez-Lavin [1] reports "an exaggerated sympathetic activity [...] described without exception [...] in women", associated with a "deficient sympathetic response to different types of stressors" and abnormalities of the gene encoding catechol-O-methyl transferase in FMS. This "hyperactivity" associated with "hyporeactivity" seems to be improved by pindolol or by "sympatholytic maneuvers".

However, Russell [2] reported in 1993 that "some patients exhibited substantially elevated norepinephrine levels but the mean values for epinephrine and norepinephrine were not significantly different from controls." Several patients had reduced levels, as confirmed by the author (IJ Russell, personnal communication), who concluded that "the relevance of this apparent heterogeneity in norepinephrine levels is not yet known."

Similarly, Crofford [3], referring to two studies [4,5], reported that "Neuropeptide Y (NPY) co-localizes with norepinephrine in the sympathetic nervous system [...] elevated plasma NPY levels are seen with [...] strong sympathetic activation. Patients with FMS have significantly lower plasma NPY levels than matched control subjects. These findings have been confirmed by Clauw et al. who demonstrated significantly lower basal NPY levels in FMS patients compared with controls as well as low NPY levels after 30 minutes on tilt table." It is noteworthy that another paper on heart rate variability by Clauw and colleagues [6] suggested a "diminished sympathetic activity" in patients with FMS and chronic fatigue syndrome. Recently, Bennett [7] confronted the data suggesting that FMS patients have an increased sympathetic tone with "an inverse association between pain sensitivity and blood pressure" and considered polymorphism of the gene encoding catechol-O-methyl transferase as only a part of the fibromyalgia genetic diversity.

With respect to specific treatments, pindolol, as discussed by Martinez-Lavin, is a non-selective $\beta$-blocking agent that possesses an intrinsic sympatho-mimetic activity. It exerts effects like epinephrine or isoproterenol and its efficiency may be similar to that of the $\beta$-stimulating agent salbutamol in FMS [8].

With regard to these data, the concept of two subgroups of patients as proposed by Russell in 1993 [2] and characterized by decreased or increased sympathetic activity and norepinephrine levels could enable Martinez-Lavin's approach to co-exist with that of Clauw and Crofford, whereas the 'no exception' rule, which complicates the already problematic diagnosis of FMS, could be amended. 


\section{Is fibromyalgia a generalized sympathetic dystrophy?}

RSD is a poorly defined condition in spite of 'new' but still unclear concepts such as complex regional pain syndrome type I (RSD) and type II (causalgia).

Sympathetic dysfunction has been partly involved in pathophysiological mechanisms of FMS and RSD but, if both conditions "affect mostly females and have frequent posttraumatic onset" [1], they exhibit more differences than similarities.

Even if controversial findings have been reported on fibromyalgia traumatic triggering factors [9], FMS occurrence is not unusual after whiplash injury. FMS is associated with subtle and diverse hormonal disorders [3], energy metabolism impairment as well as neurochemical abnormalities, such as elevated cerebrospinal fluid levels of nerve growth factor $[2,7]$. All these findings are less frequent and less marked in RSD [10] and nerve growth factor is probably increased in complex regional syndrome type II only [11].

RSD is usually observed after peripheral trauma and is perpetuated by a biphasic evolution, with a 'warm' period (biological, X-rays and isotopic findings support increased osteolysis [12]) often followed by a 'cold' period associated with trophic disorders. Spectacular improvements are sometimes reported after antiosteolytic treatment, such as biphosphonates. None of these findings is observed in fibromyalgia and the only common treatment could be calcitonin [12] and probably some antidepressants (with higher dosages in RSD).

The links between sympathetic activity and RSD have been recently reviewed by Pham and Lafforgue [10], who report that "findings suggest a decreased sympathetic tone, reduced level of norepinephrine and neuropeptide $Y$ in the affected limb..." whereas "there is a hyper reactivity to catecholamines associated with increased adrenoceptors..." Similarly, Berthelot and colleagues [11] consider that "the pathogenic role of adrenergic sympathetic activity has been successfully challenged" in this condition and that "any reference to the sympathetic system is inappropriate".

\section{Conclusion}

All these findings do not support a particular link between FMS and RSD; they suggest probable heterogeneity of dysautonomia features in FMS and could define subgroups of patients and, therefore, improve therapeutic approaches.

\section{Competing interests}

The author declares that they have no competing interests.

\section{References}

1. Martinez-Lavin M: Biology and therapy of fibromyalgia: Stress, the stress response system, and fibromyalgia. Arthritis Res Ther 2007, 9:216.
2. Russell IJ: A metabolic basis for fibromyalgia syndrome. In Progress in Fibromyalgia and Myofascial Pain. Edited by Vaeroy H, Merskey H. Amsterdam: Elsevier; 1993:283-307

3. Crofford LJ: The hypothalamic-pituitary-adrenal stress axis in the fibromyalgia syndrome. J Musculoskeletal Pain 1996, 4: 181-200.

4. Crofford LJ, Pillemer SR, Kalogeras KT, Cash JM, Michelson D, Kling MA, Sternberg EM, Gold PW, Chrousos GP, Wilder RL: Hypothalamic-pituitary-adrenal axis perturbations in patients with fibromyalgia. Arthritis Rheum 1994, 37:1583-1592.

5. Clauw DJ, Sabol M, Radulovic D, Wilson B, Katz P, Baraniuk J: Serum neuropeptides in patients with both fibromyalgia (FMS) and chronic fatigue syndrome (CFS). J Musculoskeletal Pain 1995, 3:79.

6. Clauw DJ, Radulovic D, Heshmat Y, Toby-Barbey J: Heart rate variability as a measure of autonomic function in patients with fibromyalgia (FM) and chronic fatigue syndrome (CFS). $J$ Musculoskeletal Pain 1995, 3:78.

7. Bennett RM: Three years later: Presidential address to Myopain 04. J Musculoskeletal Pain 2004, 12:1-12.

8. Eisinger J, Dupond JL: Should patients with fibromyalgia be doped? Rev Med Interne 1996, 17:977-978.

9. Tishler M, Levy O, Maslakov I, Bar-Chaim S, Amit-Vazina M: Neck injury and fibromyalgia - are they really associated? J Rheumato/ 2006, 33:1183-1185.

10. Pham T, Lafforgue P: Reflex sympathetic dystrophy syndrome and neuromediators. Joint Bone Spine 2003, 70:12-17.

11. Berthelot JM, Glemarec J, Guillot P, Maugars Y, Prost A: Algodystrophy (reflex sympathetic dystrophy syndrome) and causalgia: novel concepts regarding the nosology, pathophysiology, and pathogenesis of complex regional pain syndromes. Is the sympathetic hyperactivity hypothesis wrong? Rev Rhum Engl Ed 1997, 64:481-491.

12. Eisinger J, Acquaviva PC, d' Omezon $Y$, Schiano A, Recordier AM: Hydroxyprolinuria during algodystrophies. Therapeutic conclusions. Rev Rhum Osteoartic 1974, 41:455-458. 